\title{
Feasibility study of a novel pain assessment tool for improving prehospital pain management
}

\author{
M lqbal, P A Spaight, R Kane, Z Asghar, A N Siriwardena
}

Nottingham Conference Centre

$\underline{04 \text { February } 2015}$

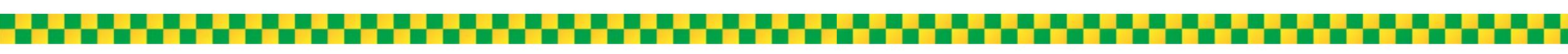

East Midlands Ambulance Service WHS 


\section{Background}

- Pain - common

- Poorly assessed

- Outcomes affected

- Previous research 


\section{Study aims}

- To develop and test a novel pain assessment tool the Patient Reported Outcome Measure for Pain Treatment (PROMPT)

- To determine feasibility, reliability and validity of the PROMPT 


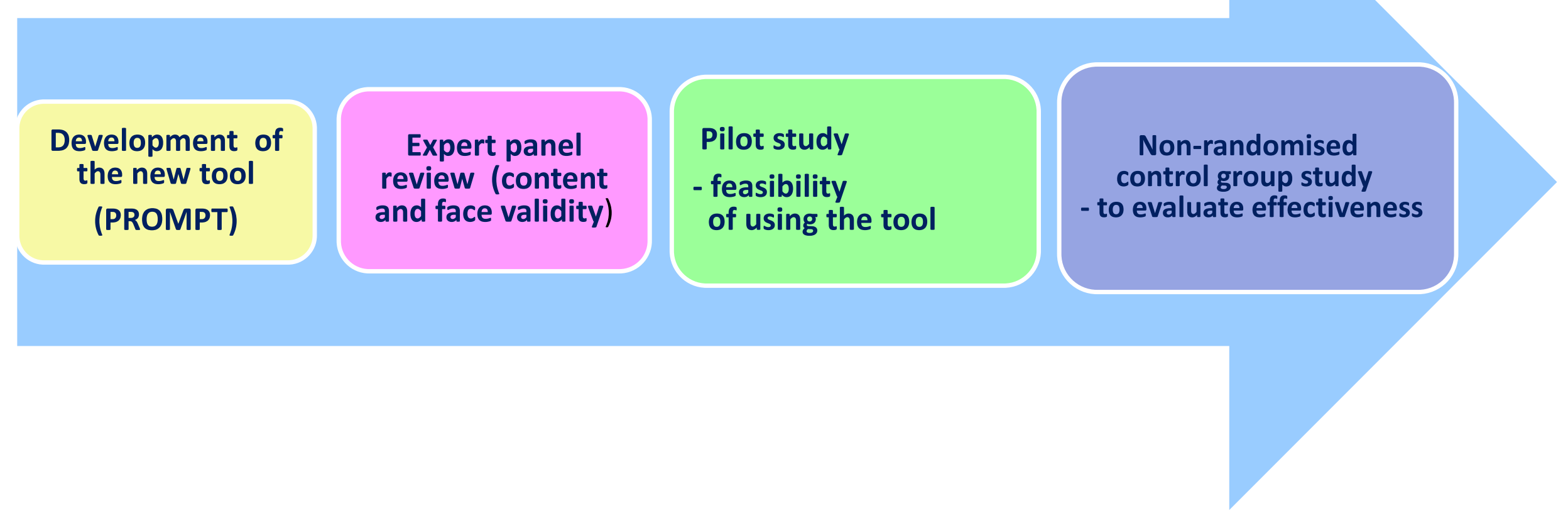




\section{Development of the tool}

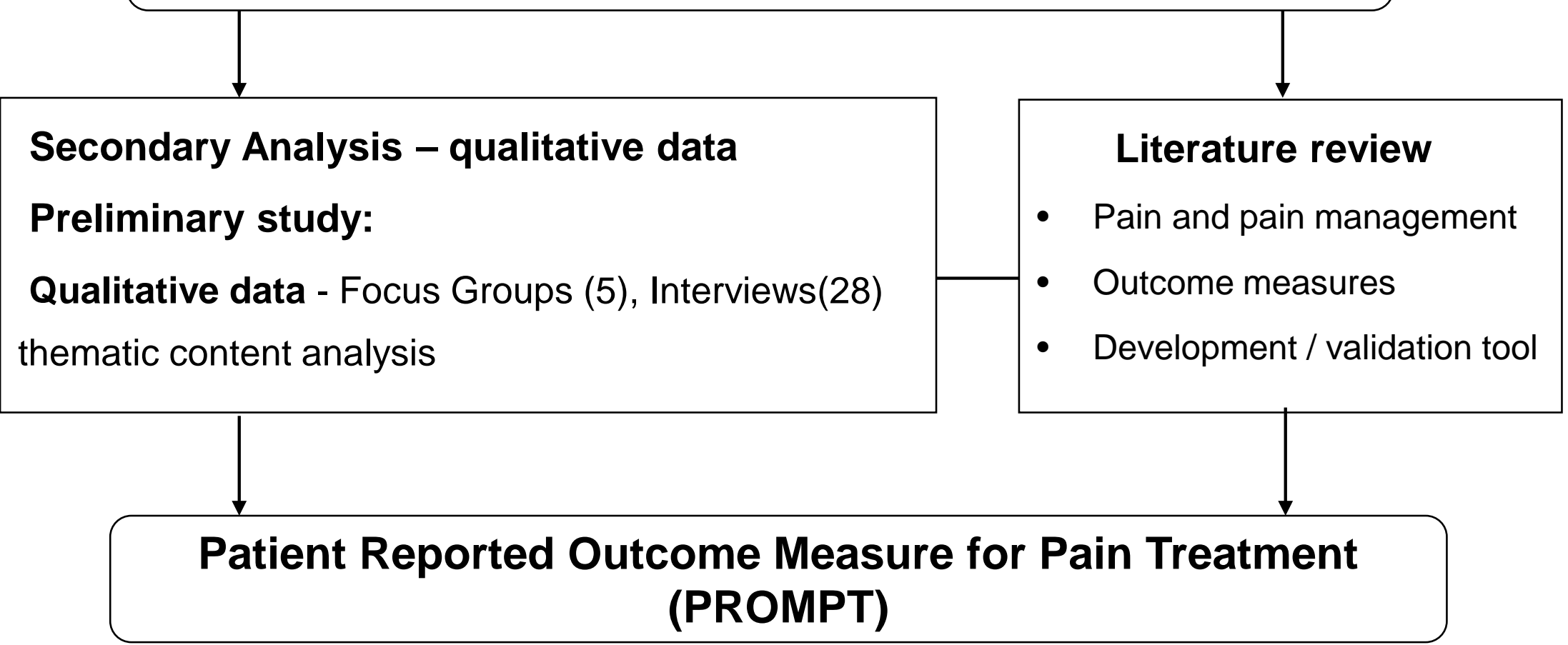




\section{Patient and practitioner Reported Outcome Measure for Pain Treatment (PROMPT)}

1.a. How much discomfort is the pain causing you?

\begin{tabular}{|c|c|c|c|c|c|}
\hline \multicolumn{5}{|c|}{ Before } \\
\hline None & Mild & Moderate & Severe & V Severe & Worst \\
\hline 0 & $1-2$ & $3-4$ & $5-6$ & $7-8$ & $9-10$ \\
\hline
\end{tabular}

2.a. How much anxiety is the pain causing you?

Before
\begin{tabular}{|c|c|c|c|c|c|}
\hline None & Mild & Moderate & Severe & V Severe & Worst \\
\hline 0 & $1-2$ & $3-4$ & $5-6$ & $7-8$ & $9-10$ \\
\hline
\end{tabular}

3.a. How much is the pain affecting your movement?

\begin{tabular}{|c|c|c|c|c|c|}
\hline \multicolumn{5}{|c|}{ Before } \\
\hline None & Mild & Moderate & Severe & V Severe & Worse \\
\hline 0 & $1-2$ & $3-4$ & $5-6$ & $7-8$ & $9-10$ \\
\hline
\end{tabular}

4.a. Pain severity (global)?

\begin{tabular}{|c|c|}
\hline \multicolumn{2}{|c|}{ Before } \\
\hline Pain score & 0 to 10 \\
\hline Patient rating & \\
\hline Clinical rating & \\
\hline
\end{tabular}

1.b. How much discomfort is the pain causing you now?

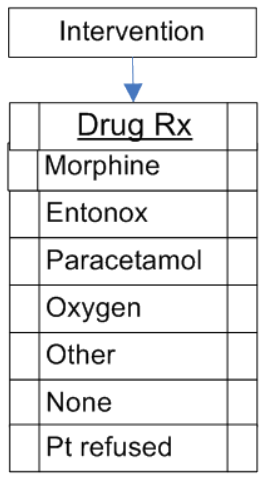

\begin{tabular}{|c|c|c|c|c|c|}
\multicolumn{9}{c|}{ After } \\
\hline None & Mild & Moderat & Severe & V Severe & Worst \\
\hline 0 & $1-2$ & $3-4$ & $5-6$ & $7-8$ & $9-10$ \\
\hline
\end{tabular}

2.b. How much anxiety is the pain causing you ne

After
\begin{tabular}{|c|c|c|c|c|c|}
\hline None & Mild & Moderate & Severe & V Severe & Worst \\
\hline 0 & $1-2$ & $3-4$ & $5-6$ & $7-8$ & $9-10$ \\
\hline
\end{tabular}

3.b. How much is the pain affecting your movement now?

\begin{tabular}{|l|l|}
\hline \multicolumn{2}{|c|}{ Intervention } \\
\hline
\end{tabular}
After

\begin{tabular}{|c|c|c|c|c|c|}
\multicolumn{7}{|c|}{ After } \\
\hline None & Mild & Moderate & Severe & V Severe & Worst \\
\hline 0 & $1-2$ & $3-4$ & $5-6$ & $7-8$ & $9-10$ \\
\hline
\end{tabular}

4.b. Pain severity (global)?

\begin{tabular}{|c|c|}
\hline \multicolumn{2}{|c|}{ After } \\
\hline Pain score & $\mathbf{0}$ to 10 \\
\hline Patient rating & \\
\hline Clinical rating & \\
\hline
\end{tabular}




\section{Expert panel review}

Panel member selected
- EMAS Clinicians - experience and length of service $>5$ years
- PROMPT, review form
Review Pack - sent (18 EMAS clinicians)

$\sqrt{ }$

Reply received - 10

$\sqrt{2}$

Analysis -

$\checkmark$ Face validity

$\checkmark$ Content validity

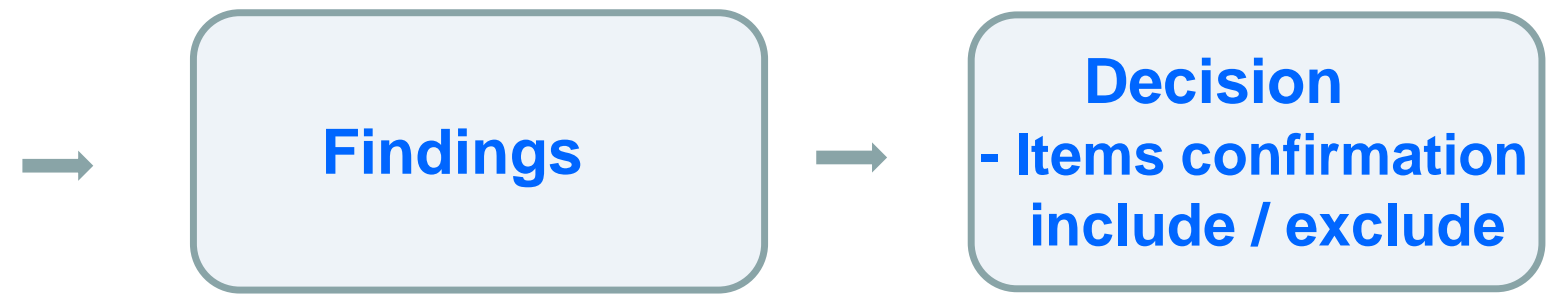

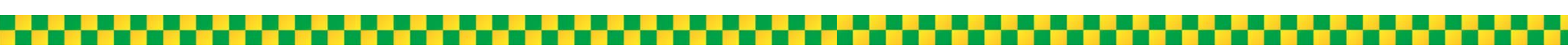
East Midlands Ambulance Service WHS 


\section{Expert panel review findings 1}

\section{Section A -}

appropriateness, practical, clarity, ambiguity, layout and workable state of the tool

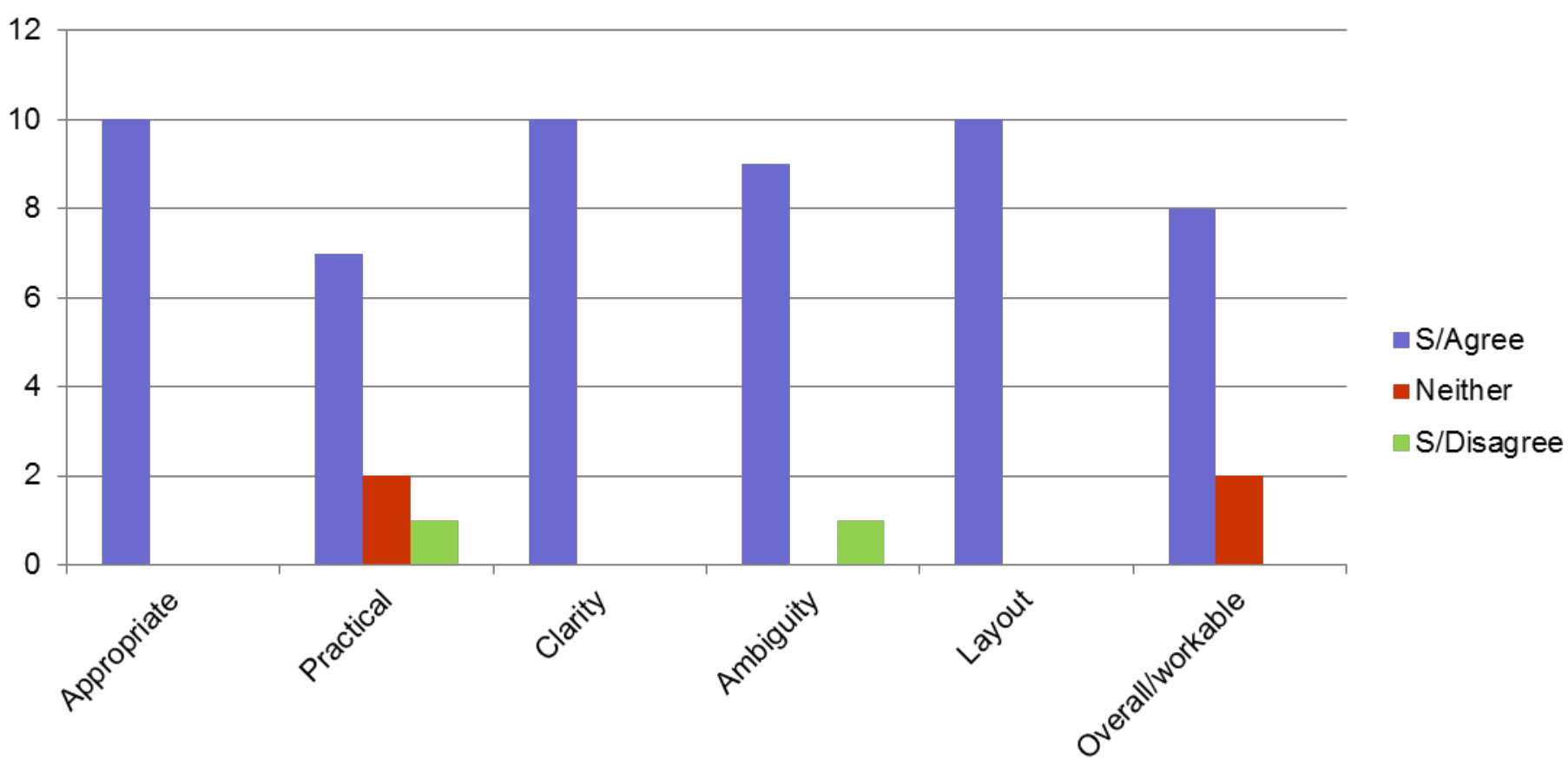




\section{Expert panel review findings 2}

- Lawshe's Content Validity Ratio (CVR) Methodology

$\begin{array}{ccc}\text { - Formula } & n_{e}-N / 2 & n_{e}=\text { number of panel members rating an item - "essential" } \\ N / 2 & N=\text { total number of panel members }\end{array}$

- CVR closer to $+1 \rightarrow$ item more essential

- CVR closer to $-1 \rightarrow$ more non-essential

- CVR - all items close to (+)1

except PS by clinician's rating weak $(-0.4)$ 
1.a. How much discomfort is the pain causing you?

\begin{tabular}{|c|c|c|c|c|c|}
\hline Before \\
\hline None & Mild & Moderate & Severe & V Severe & Worst \\
\hline 0 & $1-2$ & $3-4$ & $5-6$ & $7-8$ & $9-10$ \\
\hline
\end{tabular}

2.a. How much anxiety is the pain causing you?

\begin{tabular}{|c|c|c|c|c|c|}
\hline Before \\
\hline None & Mild & Moderate & Severe & V Severe & Worst \\
\hline 0 & $1-2$ & $3-4$ & $5-6$ & $7-8$ & $9-10$ \\
\hline
\end{tabular}

3.a. How much is the pain affecting your movements?

\begin{tabular}{|c|c|c|c|c|c|}
\hline \multicolumn{1}{|c|}{ Before } \\
\hline None & Mild & Moderate & Severe & V Severe & Worst \\
\hline 0 & $1-2$ & $3-4$ & $5-6$ & $7-8$ & $9-10$ \\
\hline
\end{tabular}

4.a. Pain severity (global)?

\begin{tabular}{|c|c|}
\hline \multicolumn{2}{|c|}{ Before } \\
\hline Pain score & 0 to 10 \\
\hline Patient rating & \\
\hline
\end{tabular}

1.b. How much discomfort is the pain causing you no

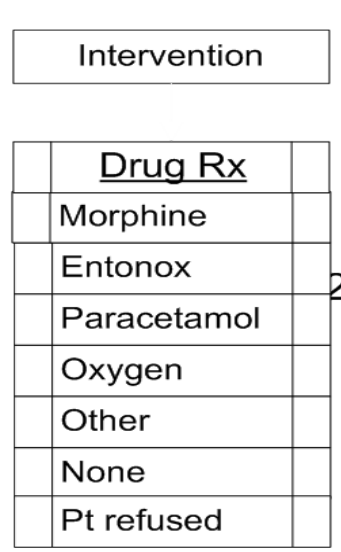

\begin{tabular}{|c|c|c|c|c|c|}
\hline \multicolumn{1}{|c|}{ After } \\
\hline None & Mild & Moderat & Severe & V Severe & Worst \\
\hline 0 & $1-2$ & $3-4$ & $5-6$ & $7-8$ & $9-10$ \\
\hline
\end{tabular}

2.b. How much anxiety is the pain causing you now?

\begin{tabular}{|c|c|c|c|c|c|}
\multicolumn{7}{c|}{ After } \\
\hline None & Mild & Moderate & Severe & V Severe & Worst \\
\hline 0 & $1-2$ & $3-4$ & $5-6$ & $7-8$ & $9-10$ \\
\hline
\end{tabular}

3.b. How much is the pain affecting your movements

\begin{tabular}{|c|}
\hline Intervention \\
\hline \\
\hline Non-drug $R x$ \\
\hline Reassurance \\
\hline Positioning \\
\hline Splint \\
\hline Support \\
\hline None \\
\hline Pt refused \\
\hline
\end{tabular}

\begin{tabular}{|c|c|c|c|c|c|}
\hline \multicolumn{1}{|c|}{ After } \\
\hline None & Mild & Moderate & Severe & V Severe & Worst \\
\hline 0 & $1-2$ & $3-4$ & $5-6$ & $7-8$ & $9-10$ \\
\hline
\end{tabular}

4.b. Pain severity (global)?

\begin{tabular}{|c|c|}
\hline \multicolumn{2}{|c|}{ After } \\
\hline Pain score & 0 to 10 \\
\hline Patient rating & \\
\hline
\end{tabular}




\section{Pilot study}

- Aim - to determine the feasibility, reliability and validity of the PROMPT

- Settings: East (Lincolnshire) Division of EMAS

- Participant and recruitment

- EMAS paramedics : emails \& memos

- 36 paramedics expressed interest

- 20 paramedics - took part in orientation training

- Orientation training

\section{East Midlands Ambulance Service WHS}




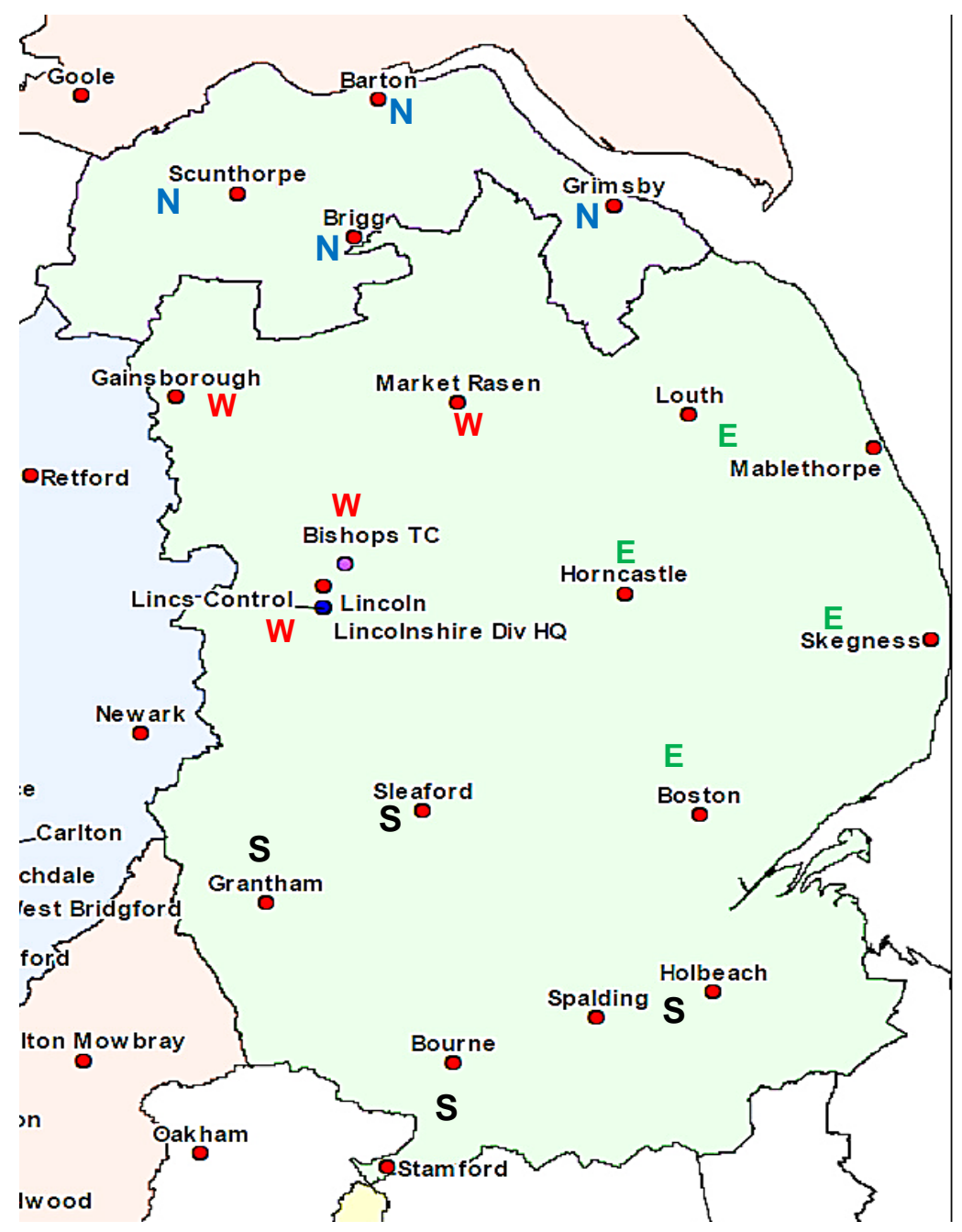

- E - East area

- W - West area

- $\quad$ - North area

- S - South area

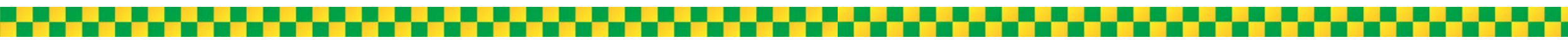 East Midlands Ambulance Service WHS}




\section{Data collection and analysis}

- Pilot data

- 18 paramedics - finally participated

- 146 completed data forms

- 132 had electronic data

- Baseline data

- 3 months routine clinical data (from electronic records)

- Analysis

- data entered in SPSS for analysis (comparing change in pain score and use of analgesic using regression)

\section{East Midlands Ambulance Service W/HS}




\section{Results}

- Cronbach's alpha $>0.8$

- Spearman's correlation 0.81 before and 0.83 after treatment given for pain 
Table 1 Patient characteristics in pilot study compared with baseline

\begin{tabular}{|c|c|c|c|c|c|}
\hline & \multicolumn{2}{|c|}{$\begin{array}{r}\text { Baseline } \\
\mathrm{N}=1776\end{array}$} & \multicolumn{2}{|c|}{$\begin{array}{l}\text { Pilot study } \\
\mathrm{N}=132\end{array}$} & \multirow[t]{2}{*}{ Chi square } \\
\hline & $\mathrm{N}$ & $\%$ & $\mathrm{~N}$ & $\%$ & \\
\hline \multicolumn{6}{|l|}{ Variables } \\
\hline \multicolumn{6}{|l|}{ Age } \\
\hline up to 20 & 49 & $(2.8)$ & 6 & $(4.5)$ & $P=0.24$ \\
\hline 21 to 40 & 259 & (14.6) & 17 & (12.9) & \\
\hline 41 to 60 & 394 & (22.2) & 37 & $(28.0)$ & \\
\hline 61 to 80 & 562 & (31.6) & 44 & (33.3) & \\
\hline over 80 & 495 & $(27.9)$ & 28 & $(21.2)$ & \\
\hline \multicolumn{6}{|l|}{ Sex } \\
\hline Male & 885 & $(49.8)$ & 56 & $(42.4)$ & $P=0.10$ \\
\hline \multicolumn{6}{|c|}{ Clinical conditions } \\
\hline Chest pain & 732 & $(41.2)$ & 73 & $(55.3)$ & $P=0.002$ \\
\hline Injury/Trauma & 1044 & $(58.8)$ & 59 & $(44.7)$ & \\
\hline
\end{tabular}

Totals are less than $100 \%$ due to missing data

\section{East Midlands Ambulance Service WHS}


Table 2 Analgesics use comparing pilot with baseline

\begin{tabular}{|c|c|c|c|c|c|}
\hline & \multicolumn{2}{|c|}{$\begin{array}{l}\text { Baseline } \\
(\mathrm{N}=1776)\end{array}$} & \multicolumn{2}{|c|}{$\begin{array}{l}\text { Pilot study } \\
(\mathrm{N}=132)\end{array}$} & \multirow[t]{2}{*}{$\mathbf{P} *$} \\
\hline & $\mathrm{N}$ & $\%$ & $\mathrm{~N}$ & $\%$ & \\
\hline Analgesic & 574 & (32.3) & 85 & (64.4) & $<0.001$ \\
\hline Morphine & 353 & (19.9) & 51 & (38.6) & \\
\hline Entonox & 262 & (14.8) & 42 & (31.8) & \\
\hline Paracetamol & 74 & (4.2) & 25 & (18.9) & \\
\hline
\end{tabular}

* Taking into account age, sex and clinical condition 
Table 3 Outcome of pain score following intervention for pain management comparing pilot with baseline

\begin{tabular}{|c|c|c|c|c|c|}
\hline & \multicolumn{2}{|c|}{$\begin{array}{l}\text { Baseline } \\
\mathrm{N}=1776\end{array}$} & \multicolumn{2}{|c|}{$\begin{array}{l}\text { Pilot study } \\
\quad \mathrm{N}=132\end{array}$} & \multirow[t]{2}{*}{$\mathbf{P} *$} \\
\hline & $\mathbf{N}$ & $\%$ & $\mathbf{N}$ & $\%$ & \\
\hline \multicolumn{6}{|c|}{ Change in pain score } \\
\hline Decreased & 614 & (34.6) & 113 & (85.6) & \\
\hline Increased & 54 & (3.0) & 1 & $(0.7)$ & $P<0.001$ \\
\hline No change & 531 & (29.9) & 18 & (13.6) & \\
\hline
\end{tabular}

Totals are less than $100 \%$ due to missing data

* Taking into account age, sex and clinical condition 


\section{Conclusions}

PROMPT : reliable and feasible with content and predictive validity

\section{Next steps}

Non-randomised control group study investigating effectiveness PROMPT compared with TAU in reduction of pain in people presenting with chest pain or injury/trauma 


\section{Acknowledgements}

Patient and Paramedic - participants

Panel members, Clinical Quality Managers, Team Leaders - EMAS

References:

1 Joint Royal Colleges Ambulance Liaison Committee, Ambulance Service Association. UK Ambulance Service Clinical Practice Guidelines. London: Ambulance Service Association, 2006

2 McLean SA, Maio RF, Domeier RM. The epidemiology of pain in the prehospital setting. Prehosp Emerg Care 2002;6: 402-405

3 Siriwardena AN, Shaw D, Bouliotis G. Exploratory cross sectional study of factors associated with prehospital management of pain. J Eval Clin Pract 2010

4 Wilson FR, Pan W and Schumsky DA. Recalculation of the Critical Values for Lawshe's Content Validity Ratio: Measurement and Evaluation in Counseling and Development 2012 45: 197 originally published online 14 March

THANK YOU!

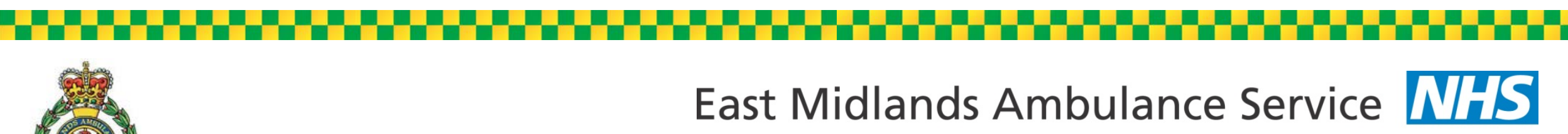

